\title{
Association between the CYP11 family and six cancer types
}

\author{
ZIWEI FAN ${ }^{1,2}$, ZHEN WANG $^{2}$, WEIRAN CHEN ${ }^{1,2}$, ZHIWEI CAO ${ }^{1}$ and YIXUE LI $^{1-3}$ \\ ${ }^{1}$ School of Life Science and Technology, Tongji University, Shanghai 200092; ${ }^{2}$ Key Lab of Computational Biology, \\ CAS-MPG Partner Institute for Computational Biology, Shanghai Institute for Biological Sciences, \\ Chinese Academy of Sciences, Shanghai 200031; ${ }^{3}$ Shanghai Center for Bioinformation Technology, \\ Shanghai Industrial Technology Institute, Shanghai 201203, P.R. China
}

Received March 11, 2015; Accepted March 22, 2016

DOI: $10.3892 / \mathrm{ol} .2016 .4567$

\begin{abstract}
Cytochromes P450 (CYPs) are a major source of variability in pharmacokinetics and drug response. CYPs utilize a variety of small and large molecules as substrates in enzymatic reactions. The CYP genes may be divided into two groups: Endogenous CYPs (CYP family 7-51) and xenobiotic CYPs (CYP family 1-4). The aim of the present study was to investigate whether endogenous CYPs exhibit similar gene expression and mutations in various cancer types. The gene expression profiles and somatic mutations exhibited in colon adenocarcinoma, kidney renal clear cell carcinoma, liver hepatocellular carcinoma, lung squamous cell carcinoma, prostate adenocarcinoma and uterine corpus endometrial carcinoma were analyzed using data obtained from The Cancer Genome Atlas. The expression of CYP11A1 was significantly downregulated in all six cancer types. In addition, CYP11B1 and CYP11B2 exhibited the highest number of mutations among endogenous CYPs in all samples. As the CYP11 family is important for steroid biosynthesis, and previous studies have demonstrated that steroid hormones are associated with certain cancers, these results indicate a common role of the CYP11 family in various cancer types.
\end{abstract}

\section{Introduction}

Cytochromes P450 (CYPs) belong to a superfamily of proteins that contain a heme cofactor. They are primarily located in the inner membrane of mitochondria or the endoplasmic reticulum of cells (1). CYPs, as the oxidase enzymes in electron transfer chains, catalyze a number of enzymatic reactions involving small molecules. The CYPs are classified as endogenous CYPs or xenobiotic CYPs. The endogenous CYPs are involved with

Correspondence to: Professor Yixue Li, Key Lab of Computational Biology, CAS-MPG Partner Institute for Computational Biology, Shanghai Institute for Biological Sciences, Chinese Academy of Sciences, 320 Yueyang Road, Shanghai 200031, P.R. China E-mail: yxli@sibs.ac.cn

Key words: cytochromes P450, CYP11 family, cancer, steroid hormones, steroid biosynthesis the biosynthesis or catabolism of steroids, sterols, retinoids, prostaglandins and fatty acids, while the xenobiotic CYPs function to defend against environmental toxins and carcinogens (2). The human CYP family 1-4 includes the major enzymes involved in drug metabolism, accounting for $\sim 75 \%$ of the total CYPs (3).

Recently, CYP studies have increasingly focused on drug metabolism (3). However, the systematic association between endogenous CYPs and cancer remains unclear. Thus, the aim of the present study was to investigate the association between endogenous CYPs and cancer. Using data obtained from The Cancer Gene Atlas (TCGA), the gene expression profiles and somatic mutations of endogenous CYPs were analyzed in six cancer types in order to determine whether any common features may exist.

In the human body, the CYP11 gene family, including CYP11A1, CYP11B1 and CYP11B2, is one of the families of CYP genes involved in steroid biosynthesis (2). Previously, almost no research has been conducted with regards to the CYP11 family and cancer; therefore, the present study aimed to investigate the associations between them. The results of the present study may be important for expanding the global view of cancer research.

\section{Materials and methods}

TCGA. Data was obtained from TCGA (http://cancergenome. nih.gov/) (4). Data regarding six diverse cancer types, including colon adenocarcinoma (COAD), kidney renal clear cell carcinoma (KIRC), liver hepatocellular carcinoma (LIHC), lung squamous cell carcinoma (LUSC), prostate adenocarcinoma (PRAD) and uterine corpus endometrial carcinoma (UCEC), were selected for analysis, to cover cancer types belonging to digestive system, respiratory system and reproductive system. The data set included 2,754 gene expression samples and 1,461 somatic mutation samples (Table I). The gene expression samples were further divided into two groups, which included 2,450 carcinoma and 304 pericarcinoma tissue samples.

Gene expression analysis. Endogenous CYPs were selected from the gene expression profile. The Wilcoxon signed-rank test was performed to analyze the differential expression of CYPs between carcinoma and pericarcinoma tissue for each cancer type. 
Table I. Expression and mutation of 56 genes in six cancer types.

\begin{tabular}{lcccc}
\hline $\begin{array}{l}\text { Cancer } \\
\text { type }\end{array}$ & $\begin{array}{c}\text { Cancer } \\
\text { samples, } \mathrm{n}\end{array}$ & $\begin{array}{c}\text { Normal } \\
\text { samples, } \mathrm{n}\end{array}$ & $\begin{array}{c}\text { Mutated } \\
\text { samples, } \mathrm{n}\end{array}$ & $\begin{array}{c}\text { Endogenous CYP } \\
\text { mutations, } \mathrm{n}\end{array}$ \\
\hline COAD & 433 & 42 & 154 & 30 \\
KIRC & 516 & 73 & 417 & 70 \\
LIHC & 148 & 51 & 203 & 25 \\
LUSC & 490 & 51 & 178 & 123 \\
PRAD & 334 & 51 & 261 & 26 \\
UCEC & 529 & 36 & 248 & 275 \\
Total & 2450 & 304 & 1461 & 549 \\
\hline
\end{tabular}

CYP, cytochrome P450; COAD, colon adenocarcinoma; KIRC, kidney renal clear cell carcinoma; LIHC, liver hepatocellular carcinoma; LUSC, lung squamous cell carcinoma; PRAD, prostate adenocarcinoma; UCEC, uterine corpus endometrial carcinoma.

Somatic mutation analysis. Data regarding the somatic mutations in endogenous CYPs were obtained (Table I). Subsequently, PolyPhen-2 (http://genetics.bwh.harvard. edu/pph2/) (5) was used to evaluate the severity of these mutations. PolyPhen-2 is a tool that predicts the possible impact of an amino acid substitution on the structure and function of a human protein using physical and comparative considerations. PolyPhen-2 generates a score for every mutation, which ranges between 0 and 1 , whereby a higher score indicates a more severe mutation. Thus, the severity of mutations may be divided into three categories: Probably damaging, possibly damaging and benign. PyMOL (http://www.pymol.org/) was used to create the protein secondary structure. PyMOL is a molecular visualization system on an open-source foundation. The protein structure data of CYP11B2 was downloaded from the Research Collaboratory for Structural Bioinformatics Protein Data Bank (RCSB PDB; http://www.rcsb.org/pdb/home/home. do).

Pathway analysis. The Kyoto Encyclopedia of Genes and Genomes (KEGG) PATHWAY database (6) (http://www. kegg.jp/kegg/pathway.html) was used to identify the metabolic pathway of CYPs. KEGG presents a collection of databases that contain information regarding genomes, biological pathways, diseases, drugs and chemical substances. KEGG is utilized for bioinformatics studies, including data analysis in genomics, metagenomics and metabolomics, modeling and simulation in systems biology and translational research in drug development.

\section{Results}

CYP gene expression profile in six cancer types. To identify differences in CYP expression patterns in various cancers, the expression of various genes was analyzed. The expression of CYP11A1 was significantly downregulated in all cancer types $(\mathrm{P}<0.001)$, while the expression of CYP27B1 was significantly upregulated $(\mathrm{P}<0.001)$ (Fig. 1A).

CYP11A1 is a mitochondrial enzyme that catalyzes the conversion of cholesterol to pregnenolone. This represents the first reaction in the process of steroidogenesis in all steroid hormone-producing mammalian tissues (Fig. 1B) (7). Low expression of CYP11A1 may cause steroid biosynthesis disorders (Fig. 2).

As shown in Fig. 1A, CYP27B1 was upregulated in 5/6 of the cancer types analyzed, with the exception of KIRC. CYP27B1 is most commonly identified in the proximal tubule of the kidney and a variety of other tissues, including skin, immune cells and bone. The enzyme catalyzes the hydroxylation of calcifediol to calcitriol (the bioactive form of vitamin D) (8).

Somatic mutations in the six cancer types. In the present study, the 10 most commonly mutated genes were identified and their functional effect was predicted using PolyPhen-2. The results revealed that all members of the CYP11 family, including CYP11A1, CYP11B1 and CYP11B2, were among the 10 most commonly mutated genes. In particular, CYP11B1 and CYP11B2 exhibited the most mutations among all genes analyzed. The major function of the CYP11 family is to promote steroid biosynthesis (9). CYP11B1 is a steroid hydroxylase present in the zona glomerulosa and zona fasciculate $(10,11)$ that generates cortisol from 11-deoxycortisol and corticosterone from 11-deoxycorticosterone. CYP11B2 is a steroid hydroxylase enzyme involved in the biosynthesis of the mineralocorticoid aldosterone. The CYP11B2 protein is only expressed in the zona glomerulosa (12) of the adrenal cortex and is primarily regulated by the renin-angiotensin system (11). CYP11B2 is the only enzyme capable of synthesizing aldosterone in humans and is important for electrolyte balance and blood pressure regulation (13). CYP7A1 exhibited the second highest number of mutations among the genes studied. It also exhibited the highest number of 'probably damaging' mutations (Fig. 3A). CYP7A1 is the rate-limiting enzyme for the synthesis of bile acid from cholesterol via the classical pathway, catalyzing the formation of 7-alpha-hydroxycholesterol (14).

As the structure of CYP11B2 may be indicated in RCSB PDB, PyMOL was used to visualize somatic mutations in the structure of CYP11B2 (Fig. 3B). Approximately $80 \%$ of mutations occurred in the $\alpha$-helix region. These mutations may alter the protein structure and subsequently affect its function.

Steroid biosynthesis pathway analysis. For the expression and mutation analysis of crucial members of the CYP11 family, 
A

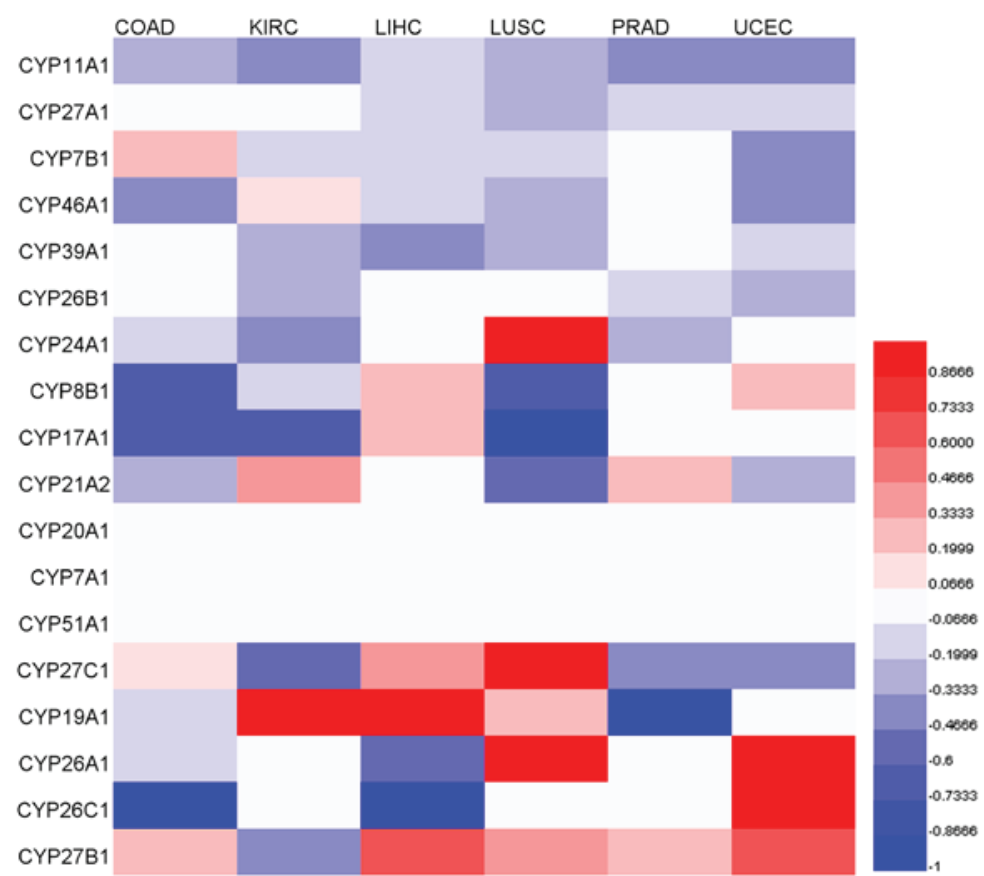

B
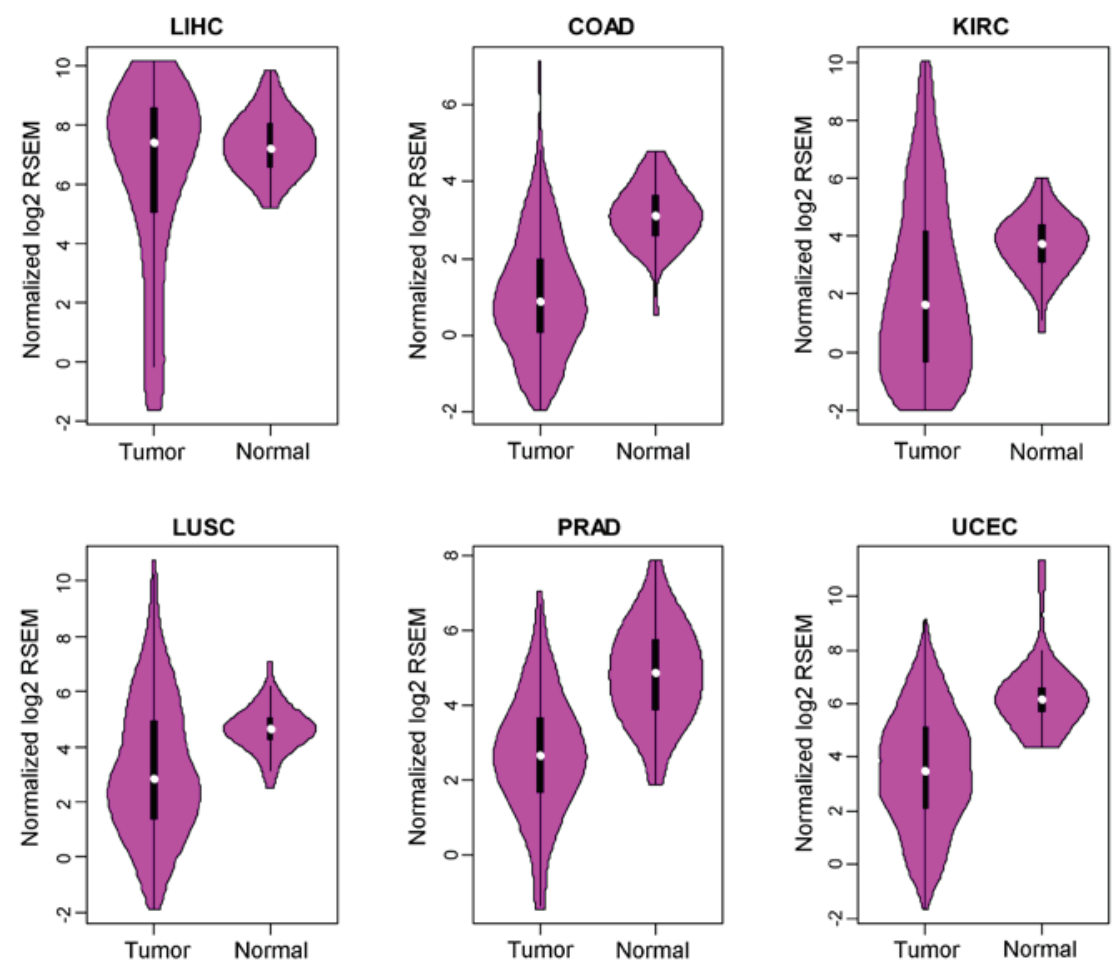

Figure 1. (A) Analysis of CYP expression in cancer and normal tissue samples showing the regulation of genes in six cancer types. A red color corresponds to upregulation and blue corresponds to downregulation. The Wilcoxon signed-rank test was used to identify significant differences in gene regulation between tumor and normal tissues. (B) Vioplot of CYP11A1 expression, showing the normalized $\log 2$ RSEM of CYP11A1 in six cancer types. CYP, cytochrome P450; COAD, colon adenocarcinoma; KIRC, kidney renal clear cell carcinoma; LIHC, liver hepatocellular carcinoma; LUSC, lung squamous cell carcinoma; PRAD, prostate adenocarcinoma; UCEC, uterine corpus endometrial carcinoma.

the KEGG pathway database was used to investigate the role of the CYP11 family in metabolic pathways. Members of the CYP11 family are involved in steroid biosynthesis. The low expression of CYP11A1 may lead to decreased expression of pregnenlone and $17 \alpha$-hydroxy pregnenolone. Furthermore, mutations in CYP11B1 and CYP11B2 may affect cortisol and aldosterone levels. Pregnenolone, cortisol and aldosterone are crucial components involved in steroid biosynthesis (Fig. 2).
Pregnenolone is a type of endogenous steroid, and is the forerunner of several steroids, including glucocorticoids, mineralocorticoids, progestogens, estrogens and androgens (15). Furthermore, pregnenolone is a biologically active neurosteroid (15). Pregnenolone is synthesized from cholesterol, a transversion that requires hydroxylation at the $\mathrm{C} 20$ and $\mathrm{C} 22$ positions of the side-chain and is performed by the enzyme CYP11A1, which is located in the mitochondria 


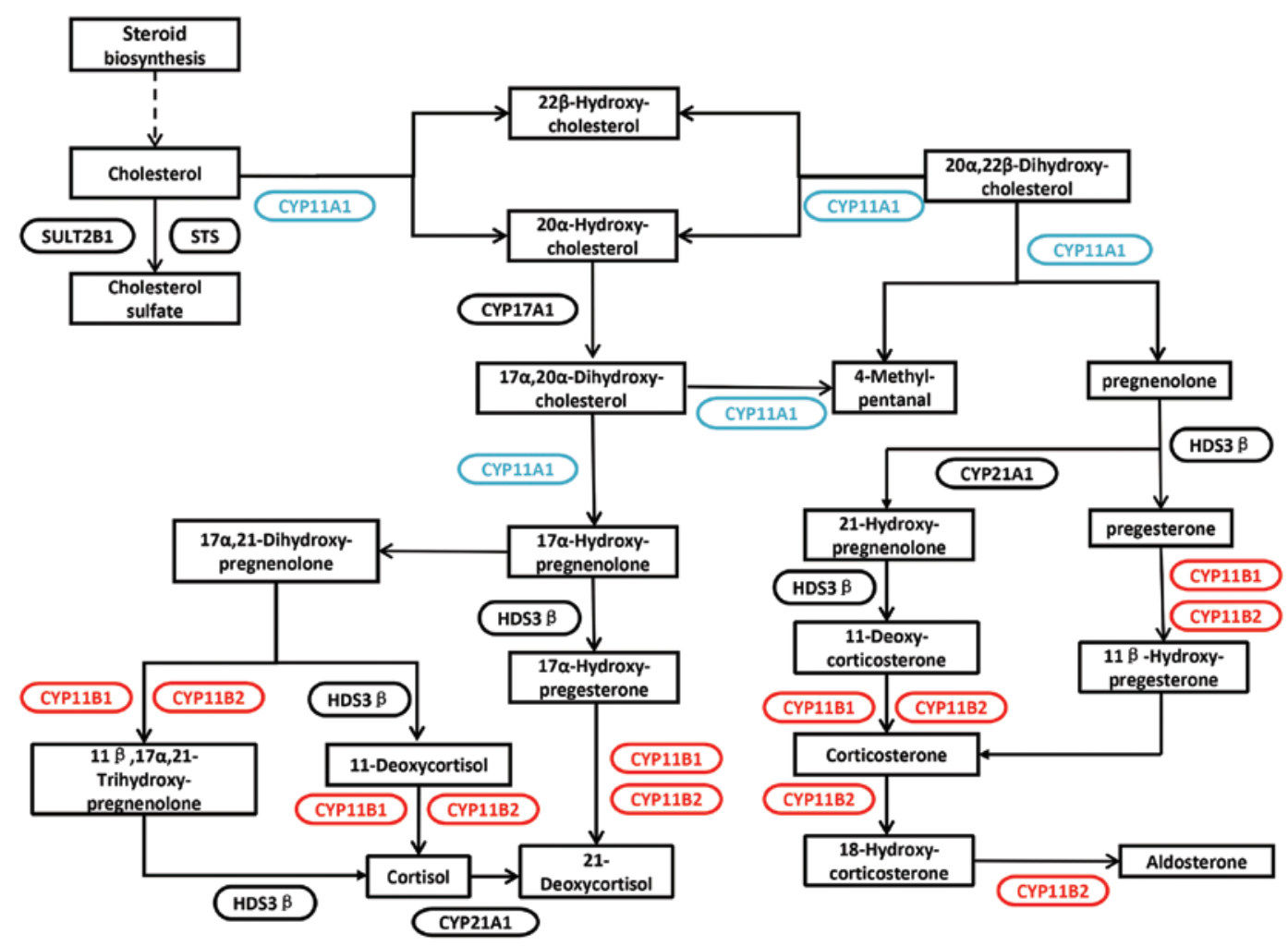

Figure 2. Steroid biosynthesis pathway. CYP11 family is involved in numerous steroid biosynthesis pathways. Blue color corresponds to downregulated expression; red color corresponds to mutation. CYP, cytochrome P450; SULT2B1, sulfotransferase family cytosolic 2B member 1; STS, sequence tagged site; HDS3 $\beta$, hydroxysteroid dehydrogenase $3 \beta$.

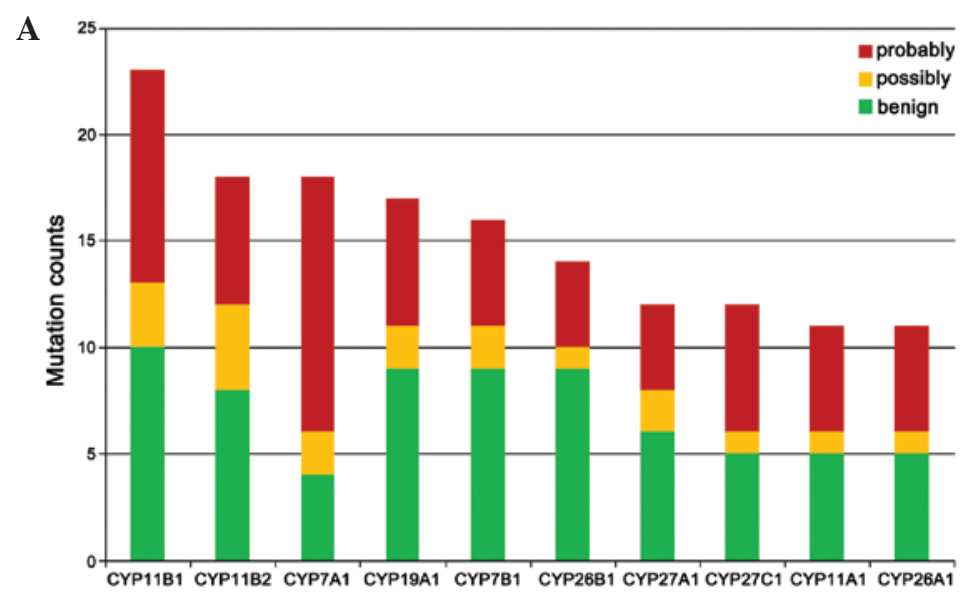

B

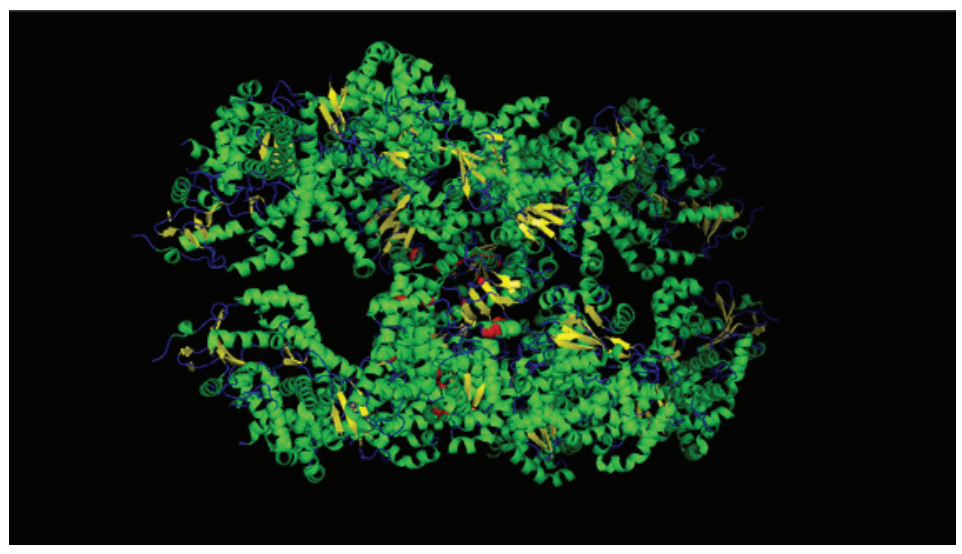

Figure 3. (A) Severity of CYP mutations were estimated in six cancer types using Polyphen-2 (http://genetics.bwh.harvard.edu/pph2/). Top 10 gene in mutation number. (B) Overall protein secondary structure of CYP11B2, created using PyMOL (http://www.pymol.org/). Green represents $\alpha$-helixes, yellow represents $\beta$-sheets, blue represents loops (neither $\alpha$-helixes nor $\beta$-sheets) and red represents mutated sequences. CYPs, cytochromes P450. 
and is controlled by anterior pituitary tropic hormones Cortisol (a glucocorticoid steroid hormone) is composed by the zona fasciculata of the adrenal cortex. During stress or hypoglycemia, cortisol will be released to suppress the immune system, to increase blood glucose, to decrease bone formation and to support the metabolism of carbohydrates, fat and protein $(16,17)$. Aldosterone (a mineralocorticoid steroid hormone) is formed by the zona glomerulosa of the adrenal cortex, and is important for blood pressure regulation (18). Blood pressure is managed by processes that occur in the distal convoluted tubules and collecting ducts of the nephron, which encourage the reabsorption of ions and water, the secretion of potassium, the conservation of sodium, and the increase in water retention, blood volume and blood pressure (18).

\section{Discussion}

The present study revealed that low CYP11A1 expression is common in several cancers, and CYP11B1 and CYP11B2 exhibit the highest number of mutations in these cancers. Approximately $80 \%$ of these mutations may alter the function of the CYP11B1 and CYP11B2 proteins. The findings of the present study indicate that the CYP11 family is commonly involved with a wide variety of cancers.

Decreased expression and mutations of the CYP11 family may influence the biosynthesis of steroid hormones. In recent years, the majority of studies of steroid hormones have been performed in breast cancer patients (19-22). However, studies have investigated the role of steroid hormones in prostate (23), lung (24), endometrial (25), colon (26) and liver cancer (27). Steroid hormones have been demonstrated to activate focal adhesion kinase, which regulates early actin reorganization in colon cancer cells (28). Steroid hormones were not previously considered to be involved with lung function (29); however, numerous studies have reported that steroid hormones are important in normal lung development and function (30) and in the pathogenesis of pulmonary diseases, including lung cancer (31-33). A study of prostate cancer validated the hypothesis that the biosynthesis of steroid hormones downstream of CYPs contributes to the progression of castration-resistant prostate cancer (34). Steroid hormones may also be utilized for the treatment of endometrial cancer. For example, progestin therapy has been demonstrated as a viable treatment option for type 1 endometrial cancer (35). These studies support the results of the present study, which indicated that steroid hormones are extremely important in numerous cancer types. In conclusion, the CYP11 family, which may affect steroid biosynthesis, is commonly involved in various types of cancer. The present study provides novel ideas that indicate that, with the development of technology, the CYP11 family could used as biomarker or drug target in the future research of cancers. Additional data and experiments will help to determine whether the genes of CYP11 family may be used as biomarkers in the diagnosis of various types of cancer. The method of computer-aided drug design may be used to simulate the interaction between the CYP11 family and chemical molecules, which will verify whether the CYP11 family could become drug targets.

\section{Acknowledgements}

The present study was supported by the National Basic Research Program of China (grant nos. 2011CB910204, 2011CB510102 and 2010CB529200) and the National Key Scientific Instrument and Equipment Development Project (grant no. 2012YQ03026108), the National Key Technology Support Program (grant no. 2013BAI101B09), and the Strategic Priority Research Program of the Chinese Academy of Sciences (grant no. XDA12000000).

\section{References}

1. Berka K, Hendrychová T, Anzenbacher P and Otyepka M: Membrane position of ibuprofen agrees with suggested access path entrance to cytochrome P450 2C9 active site. J Phys Chem A 115: 11248-11255, 2011.

2. Thomas JH: Rapid birth-death evolution specific to xenobiotic cytochrome P450 genes in vertebrates. PLoS Genet 3: e67, 2007.

3. Guengerich FP: Cytochrome p450 and chemical toxicology. Chem Res Toxicol 21: 70-83, 2008.

4. Cancer Genome Atlas Research Network; Kandoth C, Schultz N, Cherniack AD, Akbani R, Liu Y, Shen H, Robertson AG, Pashtan I, Shen R, Benz CC, et al: Integrated genomic characterization of endometrial carcinoma. Nature 497: 67-73, 2013.

5. Adzhubei I, Jordan DM and Sunyaev SR: Predicting functional effect of human missense mutations using PolyPhen-2. Curr Protoc Hum Genet Chapter 7: Unit7.20, 2013.

6. Kanehisa M, Golo S, Hattori M, Aoki-Kinoshita KF, Itoh M, Kawashima S, Katayama T, Araki M and Hirakawa M: From genomics to chemical genomics: New developments in KEGG. Nucleic Acids Res 34: D354-D357, 2006.

7. Hanukoglu I: Steroidogenic enzymes: Structure, function, and role in regulation of steroid hormone biosynthesis. J Steroid Biochem Mol Biol 43: 779-804, 1992.

8. Payne AH and Hales DB: Overview of steroidogenic enzymes in the pathway from cholesterol to active steroid hormones. Endocr Rev 25: 947-970, 2004.

9. Moon JY, Moon MH, Kim KT, Jeong DH, Kim YN, Chung BC and Choi MH: Cytochrome P450-mediated metabolic alterations in preeclampsia evaluated by quantitative steroid signatures. J Steroid Biochem Mol Biol 139: 182-191, 2014.

10. Helmberg A: Twin genes and endocrine disease: CYP21 and CYP11B genes. Acta Endocrinol (Copenh) 129: 97-108, 1993.

11. Stowasser M, Gunasekera TG and Gordon RD: Familial varieties of primary aldosteronism. Clin Exp Pharmacol Physiol 28: 1087-1090, 2001

12. Alvarez-Madrazo S, Mackenzie SM, Davies E, et al: Common polymorphisms in the CYP11B1 and CYP11B2 genes: Evidence for a digenic influence on hypertension. Hypertension 61: 232-239, 2013.

13. Padmanabhan N, Padmanabhan S and Connell JM: Genetic basis of cardiovascular disease - the renin-angiotensin-aldosterone system as a paradigm. J Renin Angiotensin Aldosterone Syst 1: 316-324, 2000.

14. Davis RA, Miyake JH, Hui TY and Spann NJ: Regulation of cholesterol-7alpha-hydroxylase: BAREly missing a SHP. J Lipid Res 43: 533-543, 2002.

15. Marx CE, Bradford DW, Hamer RM, Naylor JC, Allen TB, Lieberman JA, Strauss JL and Kilts JD: Pregnenolone as a novel therapeutic candidate in schizophrenia: Emerging preclinical and clinical evidence. Neuroscience 191: 78-90, 2011.

16. Hoehn K and Marieb EN (eds): The endocrine system. In: Human Anatomy \& Physiology. 8th edition. Benjamin Cummings, San Francisco, CA, pp606-612, 2010.

17. Chyun YS, Kream BE and Raisz LG: Cortisol decreases bone formation by inhibiting periosteal cell proliferation. Endocrinology 114: 477-480, 1984.

18. Hu C, Rusin CG, Tan Z, Guagliardo NA and Barrett PQ: Zona glomerulosa cells of the mouse adrenal cortex are intrinsic electrical oscillators. J Clin Invest 122: 2046-2053, 2012.

19. Claus EB, Risch N and Thompson WD: Genetic analysis of breast cancer in the cancer and steroid hormone study. Am J Hum Genet 48: 232-242, 1991.

20. He Q, Liang CH and Lippard SJ: Steroid hormones induce HMG1 overexpression and sensitize breast cancer cells to cisplatin and carboplatin. Proc Natl Acad Sci USA 97: 5768-5772, 2000. 
21. Colleoni M, Viale G, Zahrieh D, et al: Chemotherapy is more effective in patients with breast cancer not expressing steroid hormone receptors: A study of preoperative treatment. Clin Cancer Res 10: 6622-6628, 2004.

22. Eliassen AH, Missmer SA, Tworoger SS, Spiegelman D, Barbieri RL, Dowsett $M$ and Hankinson SE: Endogenous steroid hormone concentrations and risk of breast cancer among premenopausal women. J Natl Cancer Inst 98: 1406-1415, 2006.

23. Platz EA, Leitzmann MF, Rifai N, Kantoff PW, Chen YC, Stampfer MJ, Willett WC and Giovannucci E: Sex steroid hormones and the androgen receptor gene CAG repeat and subsequent risk of prostate cancer in the prostate-specific antigen era. Cancer Epidemiol Biomarkers Prev 14: 1262-1269, 2005.

24. Kaiser U, Hofmann J, Schilli M, Wegmann B, Klotz U, Wedel S, Virmani AK, Wollmer E, Branscheid D, Gazdar AF and Havemann K: Steroid-hormone receptors in cell lines and tumor biopsies of human lung cancer. Int J Cancer 67: 357-364, 1996.

25. Potischman N, Hoover RN, Brinton LA, Siiteri P, Dorgan JF, Swanson CA, Berman ML, Mortel R, Twiggs LB, Barrett RJ, et al: Case-control study of endogenous steroid hormones and endometrial cancer. J Natl Cancer Inst 88: 1127-1135, 1996.

26. Lointier P, Wildrick DM and Boman BM: The effects of steroid hormones on a human colon cancer cell line in vitro. Anticancer Res 12: 1327-1330, 1992

27. De Maria N, Manno M and Villa E: Sex hormones and liver cancer. Mol Cell Endocrinol 193: 59-63, 2002.
28. Gu S, Kounenidakis M, Schmidt EM, Deshpande D, Alkahtani S, Alarifi S, Föller M, Alevizopoulos K, Lang F and Stournaras C: Rapid activation of FAK/mTOR/p70S6K/PAK1-signaling controls the early testosterone-induced actin reorganization in colon cancer cells. Cell Signal 25: 66-73, 2013.

29. Koehler KF, Helguero LA, Haldosén LA, Warner M and Gustafsson JA: Reflections on the discovery and significance of estrogen receptor beta. Endocr Rev 26: 465-478, 2005.

30. Patrone C, Cassel TN, Pettersson K, Piao YS, Cheng G, Ciana P, Maggi A, Warner M, Gustafsson JA and Nord M: Regulation of postnatal lung development and homeostasis by estrogen receptor beta. Mol Cell Biol 23: 8542-8552, 2003.

31. Bardin A, Boulle N, Lazennec G, Vignon F and Pujol P: Loss of ERbeta expression as a common step in estrogen-dependent tumor progression. Endocr Relat Cancer 11: 537-551, 2004.

32. Bouchardy C, Benhamou S, Schaffar R, Verkooijen HM, Fioretta G, Schubert H, Vinh-Hung V, Soria JC, Vlastos G and Rapiti E: Lung cancer mortality risk among breast cancer patients treated with anti-estrogens. Cancer 117: 1288-1295, 2011.

33. Kazmi N, Márquez-Garbán DC, Aivazyan L, Hamilton N, Garon EB, Goodglick L and Pietras RJ: The role of estrogen, progesterone and aromatase in human non-small-cell lung cancer. Lung Cancer Manag 1: 259-272, 2012.

34. de Bono JS, Logothetis CJ, Molina A, Fizazi K, North S, Chu L, Chi KN, Jones RJ, Goodman OB Jr, Saad F, et al; COU-AA-301 Investigators: Abiraterone and increased survival in metastatic prostate cancer. N Engl J Med 364: 1995-2005, 2011.

35. Kim JJ, Kurita T and Bulun SE: Progesterone action in endometrial cancer, endometriosis, uterine fibroids, and breast cancer. Endocr Rev 34: 130-162, 2013 